\title{
Basophils make food hard to swallow
}

New research published in Nature

Medicine has led to the identification of a mechanism that is responsible for an increasingly recognized food allergy-related disease known as eosinophilic oesophagitis (EoE). Patients with EoE generally show a narrowing of the oesophagus, which leads to difficulty swallowing and food impaction. The study suggests that food allergy-associated inflammation in the oesophagus is driven by increased levels of the cytokine thymic stromal lymphopoietin (TSLP) that promote exaggerated basophil responses.

The recent finding that a gain-of-function polymorphism in the gene encoding TSLP is associated with the development of EoE led the authors to investigate the role of this cytokine in the disease. They generated a disease model in which mice were first sensitized to a food allergen - ovalbumin (OVA) through the skin, using the vitamin D analogue MC903 or tape-stripping to generate a lesion, and were then orally challenged with OVA. Similar to the disease in humans, mice that were sensitized and challenged with OVA developed inflammation and eosinophilia in the oesophagus. This EoE-like disease was associated with increased TSLP expression in the skin and oesophageal tissues, as well as with high levels of mRNA encoding

T helper 2-type cytokines and a basophil-specific protease. Further analysis showed that $30 \%$ of mice with the disease had impacted food in the oesophagus at the time of killing, whereas food impaction was never observed in control mice. To explore the involvement of TSLP in this mouse model of EoE, the authors first studied TSLP receptor (TSLPR)-deficient mice. Unlike wild-type mice, Tslpr ${ }^{-1-}$ mice did not develop oesophageal eosinophilia following sensitization and challenge with OVA. In further support of a necessary and sufficient role for TSLP, it was shown

that EoE-like disease developed after oral challenge when wild-type mice were sensitized to OVA in the presence of recombinant TSLP, but not when they were treated with OVA alone. Interestingly, although the EoE-like disease was associated with high levels of OVA-specific

targeting the TSLP-basophil pathway ... could effectively treat mice with established

EoE-like disease IgE, IgE-deficient and IgE-sufficient mice developed equivalent diseases, which suggests that the disease is $\operatorname{IgE}$ independent. This is consistent with reports that indicate that IgE-targeted therapies fail to ameliorate EoE in most patients.

TSLP is also known to promote basophil responses, which suggests that basophils are involved in EoE. Consistent with this idea, the depletion of basophils during the sensitization phase, using either diphtheria toxin-based genetic depletion or the basophil-depleting CD200R3-specific antibody, limited the development of EoE-like disease. Moreover, targeting the TSLP-basophil pathway with a neutralizing TSLP-specific antibody or a CD200R3-specific antibody could effectively treat mice with established EoE-like disease, as indicated by decreased oesophageal eosinophilia and improved oesophageal function compared with mice treated with a control antibody.

Consistent with the idea of targeting the TSLP-basophil axis to treat human disease, the authors observed increased TSLP expression and basophil frequencies in oesophageal biopsy samples from paediatric and adult patients with active EoE, compared with individuals without EoE or with inactive EoE. In addition, higher basophil frequencies were present in individuals who were homozygous or heterozygous for the riskassociated TSLP polymorphism. Taken together, these data suggest that patients with the TSLP risk allele have a predisposition to TSLP overexpression and the associated basophilia that might increase their likelihood of developing EoE after an encounter with trigger antigens.

Lucy Bird

ORIGINAL RESEARCH PAPER Noti, M. et al. Thymic stromal lymphopoietin-elicited basophil responses promote eosinophilic esophagitis. Nature Med. 19, 1005-1013 (2013) 